\title{
TEOR DE CLOROFILA, CAROTENÓIDES E ÍNDICE SPAD NA ALFACE (Lactuca sativa) EM FUNÇÃO DE LÂMINAS DE IRRIGAÇÃO E NÍVEIS SALINOS
}

\author{
Cinara Bernardo da Silva1', Julianna Catonio da Silva², Williams Querino Brandão Junior ${ }^{3}$, Floriano Alcântara \\ Damasceno ${ }^{3}$, Marcelo Rodrigues Barbosa Júnior ${ }^{5}$, Marcio Aurélio Lins dos Santos ${ }^{6}$
}

\begin{abstract}
'Engenheira Agrônoma, Mestre em Agricultura e Ambiente, Extensionista Emater/ Arapiraca-Al, Rua Governador Silvestre Péricles, № 1065, Jardim Tropical, Arapiraca/AL Brasil, CEP 57313-390

${ }^{2}$ Engenheira Agrônoma, Mestre em Agricultura e Ambiente, Doutoranda em Produção Vegetal, Universidade Federal de Alagoas, Campus de Engenharias e Ciências Agrárias BR-104, Km 85, s/n. Rio Largo - AL CEP 57100-000

${ }^{3}$ Graduando em Agronomia na Universidade Federal de Alagoas, Campus de Arapiraca, Avenida Manoel Severino Barbosa, S/N, Bom Sucesso, Arapiraca - AL, Cep: 57309-005

${ }^{4}$ Engenheiro Agrônomo, Mestrando em Produção Vegetal, Universidade Estadual Paulista, Via de Acesso Professor Paulo Donato Castellane, S/N - Vila Industrial, Jaboticabal - SP, CEP: 14884-900

${ }^{5}$ Professor Doutor da Universidade Federal de Alagoas, Campus de Arapiraca, Avenida Manoel Severino Barbosa, S/N, Bom Sucesso, Arapiraca - AL, Cep: 57309-005
\end{abstract}

RESUMO: A alface é uma folhosa sensível a salinidade da água de irrigação e ao estresse hídrico e seus danos são causados por diversos fatores inerentes a fisiologia da planta. Objetivou-se com este trabalho avaliar diferentes lâminas de água e níveis salinos e seus efeitos na cultura da alface, através do SPAD, clorofilas (a e b) e carotenoides. 0 experimento foi conduzido em casa de vegetação no Campus de Arapiraca da Universidade Federal de Alagoas. 0 delineamento experimental utilizado foi em blocos casualizados, num esquema fatorial $5 \times 5$, com 5 repetições, totalizando 125 parcelas experimentais. Os tratamentos foram representados por 5 lâminas de irrigação, correlacionadas a 5 níveis de salinidade. As variáveis analisadas foram: conteúdo de clorofila, clorofilas (a e b) e carotenoides. Os dados foram submetidos à análise de regressão com o programa estatístico SISVAR. A variável SPAD apresentou comportamento quadrático, onde a partir da lâmina de $123 \%$ atingiu 13 índice de SPAD; para carotenoides ocorreu interação dos dois fatores estudados, no qual seus efeitos influenciaram de forma positiva e negativa no desenvolvimento da alface e as clorofilas não apresentaram diferença.

PALAVRAS-CHAVE: Lactuca sativa; água; fisiologia.

\section{CHLOROPHYLL, CAROTENOIDS AND SPAD CONTENT ON LIFFACE (Lactuca sativa) AS A FUNCTION OF IRRIGATION BLADES AND SALT LEVELS}

\begin{abstract}
Lettuce is a hardwood sensitive to irrigation water salinity and water stress and its damage is caused by several factors inherent to plant physiology. The objective of this work was to evaluate different water depths and saline levels and their effects on lettuce culture through SPAD, chlorophylls ( $a$ and $b$ ) and carotenoids. The experiment was conducted in a greenhouse at Arapiraca Campus of the Federal University of Alagoas. The experimental design was randomized blocks in a $5 \times 5$ factorial scheme with 5 replications, totaling 125 experimental plots. The treatments were represented by 5 irrigation depths, correlated to 5 salinity levels. The variables analyzed were: chlorophyll content, chlorophylls ( $a$ and $b$ ) and carotenoids. Data were subjected to regression analysis with the statistical program SISVAR. The SPAD variable presented quadratic behavior, where from the $123 \%$ blade reached 13 SPAD index; For carotenoids there was interaction of the two factors studied, in which their effects positively and negatively influenced the development of lettuce and chlorophylls showed no difference.
\end{abstract}

KEYWORDS: Lactuca sativa; Water; physiology. 


\section{INTRODUÇÃO}

A cultura da alface (Lactuca sativa L.) possui característica herbácea, tem caule diminuto e não ramificados. Possui raiz pivotante com extrema ramificação, o que permite a exploração intensa na proximidade da inserção ao meio de cultivo (Filgueira, 2008). É utilizada principalmente na alimentação humana oferecendo grande importância em dietas com restrição calórica, já que a porção de 100 gramas não excede a $15 \mathrm{Kcal}$. Possui quantidades consideráveis de minerais e vitaminas A, B1, B2, B6 e C (Bezerra et al., 2006; Ribeiro, 2016).

Quando se trata da produção de hortícolas, sabe-se que está frequentemente associada à irrigação localizada, esta que apresenta uma crescente taxa de adoção entre os produtores devido à mesma possibilitar um melhor aproveitamento da água, garantindo produtividades mais elevadas. As hortaliças em sua maioria possuem $95 \%$ de água em sua constituição, onde o manejo de irrigação deve ser considerado prática importante para obtenção de alta qualidade e produtividade da cultura (Testezlaf e Matsura, 2015; Bernardo et al., 2013).

Segundo Ayers e Wostcot (1991) a alface é considerada moderadamente sensível à salinidade, sendo que seu rendimento potencial é alcançado quando a condutividade elétrica do extrato saturação atinge 0 valor liminar de 1,3 dS $\mathrm{m}^{-1}$ com redução de $13 \%$ do rendimento por aumento unitário de salinidade acima do valor limite.

Objetivou-se com este trabalho avaliar diferentes lâminas de água e níveis salinos, como também seus efeitos na cultura da alface, através do SPAD, clorofilas (a e b) e carotenoides.

\section{MATERIAL E MÉTODOS}

0 experimento foi conduzido em casa de vegetação no Campus de Arapiraca, na Universidade Federal de Alagoas, com coordenadas geodésicas $\left(09^{\circ}\right.$ $48^{\prime} 40,3^{\prime \prime}$ S e $36^{\circ} 37^{\prime} 19,7^{\prime \prime}$ O, altitude de $245 \mathrm{~m}$ ).

O solo foi classificado como LATOSSOLO VERMELHO-AMARELO Distrófico (Embrapa, 2013). Utilizou-se a variedade lisa (Lactuca sativa $\mathrm{L}$ ).

Os tratamentos foram: cinco lâminas de irrigação $L_{1}=50 ; L_{2}=75 ; L_{3}=100 ; L_{4}=125$ e $L_{5}=150 \%$ da Evapotranspiração da cultura (ETc) e cinco níveis de água salina com $\mathrm{S} 1=0,12 ; \mathrm{S} 2=1,12 ; \mathrm{S} 3=2,12 ; \mathrm{S} 4=$
3,12 e S5 = 4,12 dS m$~^{-1}$. O delineamento experimental foi em blocos ao acaso (DBC), com 5 repetições, em esquema fatorial $5 \times 5,25$ tratamentos e 125 unidades experimentais.

A água foi preparada diariamente utilizando a relação entre condutividade elétrica da água de irrigação (CEa) e concentração ( $\mathrm{mg} \mathrm{L}^{-1}=640^{*} \mathrm{CE}$ ), obtida de Richards et al. (1999). Utilizou-se para a medição da CEa um condutivímetro portátil.

Utilizou-se recipientes de plástico com capacidade para 4 litros, que foram perfurados na sua base inferior e inserido um dreno. Colocou-se ao fundo, nas perfurações, uma tela de nylon e em seguida preenchidos com $2 \mathrm{~cm}$ de brita zero, tendo por objetivo a drenagem da água evitando a passagem de sedimentos. Por fim, os recipientes foram preenchidos com $04 \mathrm{~kg}$ de solo. Os recipientes foram saturados, logo após a saturação a água foi drenada e 24 horas após o solo encontrava-se em capacidade de campo.

0 sistema de irrigação foi montado em casa de vegetação, utilizando gotejadores, um em cada recipiente. A água foi proveniente de reservatórios, localizados em uma bancada, a qual era direcionada para os recipientes através da gravidade por tubulações para um conjunto de 25 registros, sendo, cada registro responsável pela aplicação de um tratamento.

Realizou-se a aferição do tempo de vazão em cada lâmina, utilizando um recipiente plástico de volume conhecido. Após isso, obteve-se o controle do tempo da água aplicada, em seguida foi determinado a vazão do sistema de irrigação, e por fim, realizado o cálculo da irrigação utilizando uma planilha. Posteriormente ocorreu a adubação.

As irrigações ocorreram no final da tarde de cada dia, com base no consumo de água das plantas de cinco lisímetros de drenagem. Na primeira irrigação, aplicou-se $500 \mathrm{ml}$ de água por planta. Após 24 horas da aplicação nos lisímetros a água foi coletada, e esses dados foram inseridos em uma planilha eletrônica, que forneceu a irrigação a ser aplicada nos demais blocos.

Foram avaliadas as variáveis: conteúdo de clorofila utilizando o clorofilômetro SPAD-502 (Minolta Corp, Ramsey, NJ, EUA), clorofilas (a e b) e carotenoides. Os dados foram submetidos à análise de regressão com o programa estatístico SISVAR. 


\section{RESULTADOS E DISCUSSÃO}

Houve efeito significativo para fator lâmina de irrigação para o índice de clorofila SPAD. Para carotenoides houve interação entre os dois fatores estudados (Lâmina e salinidade), não sendo significativo para clorofila a e b.

De acordo com os cálculos efetuados a variável SPAD apresentou comportamento quadrático, onde a partir da lâmina de 123\% atingiu 13 índice de SPAD para a planta (Figura 1).

Figura 1. Índice de clorofila sob diferentes lâminas de

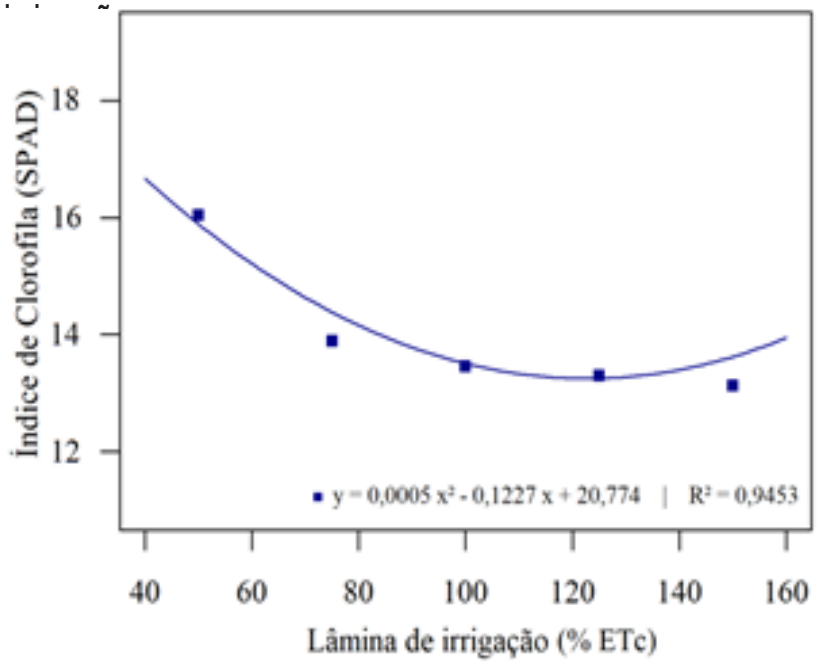

Segundo Carvalho et al. (2012), quando ocorre um aumento da leitura SPAD significa que houve uma melhor assimilação do nitrogênio pela planta. Para Winder (2018) o teor de clorofila é indicativo da quantidade de nitrogênio presente nas folhas e serve como base informativa para identificação de sua deficiência que pode ocorrer devido ao excesso de água e a lixiviação do Nitrogênio presente no solo. Sendo assim, o aumento da lâmina pode ter influenciado na lixiviação dos nutrientes, entre eles, o nitrogênio ocasionando na coloração mais claras das folhas.

Com relação a carotenoides, observou-se, no desdobramento da lâmina de irrigação dentro de cada nível salino, com modelos de equação linear crescente e cúbico, para os níveis salinos de 0,12 e 3,12 dS $\mathrm{m}^{-1}$ (Figura 2a). Conforme ocorreu 0 aumento da lâmina houve um aumento nos carotenoides, isso quando a

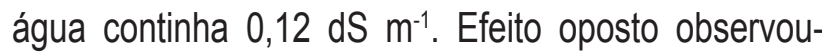
se na lâmina com maior teor de sais de 3,12 dS m $\mathrm{m}^{-1}$, ocasionando um tipo de desbalanceamento, com uma redução nas quantidades de carotenoides conforme aumentava a lâmina de irrigação.

Já para o desdobramento de salinidade dentro de cada lâmina de irrigação ajustaram-se aos modelos quadrático, cúbico e linear decrescente, para as lâminas de 50,75 e $150 \%$ da Etc. Para o modelo quadrático, correspondente a lâmina de 50\%, observou-se que a salinidade de 3,34 $\mathrm{dS} \mathrm{m}^{-1}$ proporcionou o valor máximo de carotenoides nas plantas, atingindo $0,18 \mathrm{~g} \mathrm{Kg}-1 \mathrm{de}$ carotenoides, posteriormente houve um decréscimo (Figura 2b).

Figura 2. Carotenoides em função da lâmina de irrigação $(A)$ e níveis de salinidade (B)

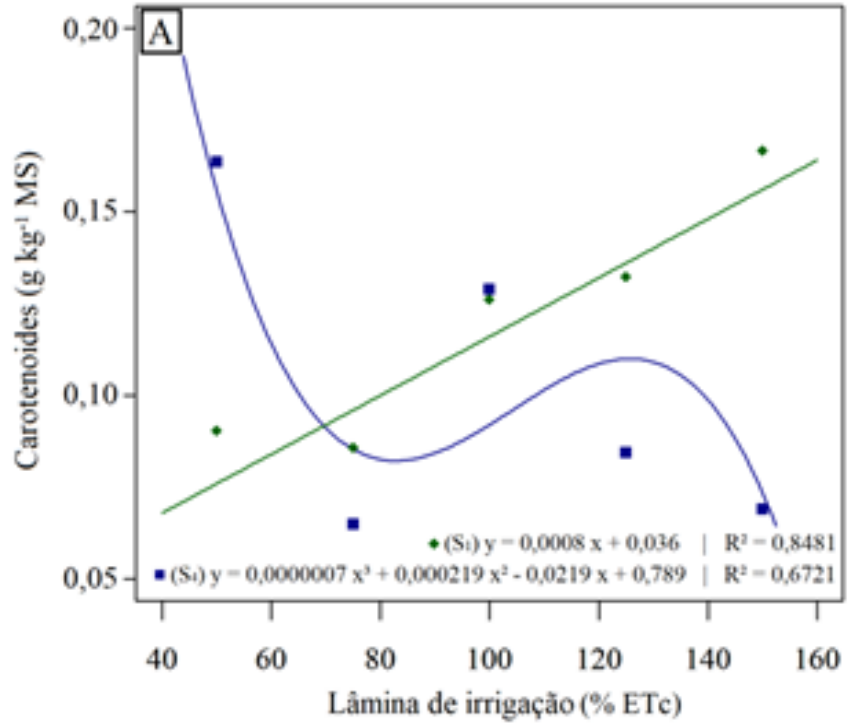

Silva et al., (2016) verificaram que houve incrementos nos teores de carotenóides à medida que

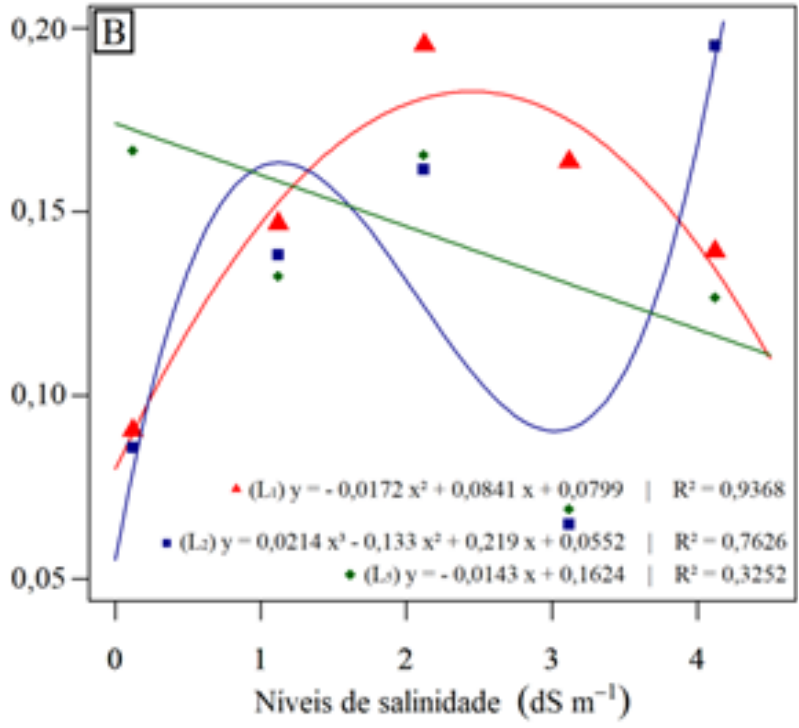

se aumentou a aplicação de água. Segundo Fiaz et al., (2014), a insuficiência de água acarreta redução na 
atividade enzimática da protoclorofila redutase, inibindo a conversão dos respectivos precursores em pigmentos fotossintéticos, sendo essa redução apontada como a principal razão da menor produção de pigmentos fotossintéticos, inclusive de carotenóides, quando as plantas são submetidas à deficiência hídrica.

Já para a salinidade, Silva et al., (2016) relatam que por outro lado, a expressão da resposta à elevação do nível de salinidade do solo apontou que os crescentes níveis de CE proporcionaram reduções no teor de carotenoides presentes nas folhas. Para Gomes et al., (2011) esse comportamento pode ser atribuído, dentre outras causas, ao fato do estresse salino desacelerar a produção de pigmentos fotossintéticos, induzindo a degradação de $\beta$ - caroteno, provocando uma diminuição no teor de carotenoides, que são componentes integrados dos tilacoides, atuantes na absorção e na transferência de luz para a clorofila.

Observou-se que o aumento da lâmina de irrigação influenciou no índice de clorofila na planta (SPAD). Já para carotenoides ocorre interação dos dois fatores estudados, no qual seus efeitos influenciaram de forma positiva e negativa no desenvolvimento da alface.

\section{REFERÊNCIAS BIBLIOGRÁFICAS}

Ayers, R. S.; Westcot, D. W. A qualidade da água na agricultura. 1991, Campina Grande: UFPB. 218p..

Bernardo, S., Soares, A. A., Mantovani, E. C. Manual de Irrigação. 2013, 8. Ed. Viçosa: Ed. UFV, p. 23-463.

Bezerra Neto, F.; Barros Júnior, A. P.; Silva, E. O.; Negreiros, M. Z.; Oliveira, E. Q.; Silveira, L. M.; Câmara, M. J. T.; Nunes, G. H. S. Qualidade nutricional de cenoura e alface cultivadas em Mossoró-RN em função da densidade populacional. Horticultura Brasileira, 2006, 24, 4, 476-480.

Carvalho, K. S.; Bonfim-Silva, E. M.; Silveira, M. H. D.; Cabral, C. E. A.; Leite, N. Rúcula submetida à adubação nitrogenada via fertirrigação. Enciclopédia Biosfera, 2012, 8, 15, 1545-1553.

Embrapa- Empresa Brasileira de Pesquisa Agropecuária. Centro Nacional de Pesquisa de Solos. 3. ed. Brasília: EMBRAPA-SPI, 2013, p.353.
FIAZ, K.; MALIK, S. A.; YOUNIS, U.; DANISH, S.; RAZA SHAH, M. H.; NIAZ, S. Drought impact on $\mathrm{Pb} / \mathrm{Cd}$ toxicity remediated by biochar in Brassica campestris. Journal of Soil Science and Plant Nutrition, 2014, 14, $4,845-854$.

Filgueira, F. A. R. Novo manual de olericultura: agrotecnologia moderna na produção e comercialização de hortaliças, 2008, Viçosa, MG: UFV, 421 p.

Gomes, M. A. da C.; Suzuki, M. S.; Cunha, M. da; Tullii, C. F. Effect of salt stress on nutrient concentration, photosynthetic pigments, proline and foliar morphology of Salvinia auriculata Aubl. Acta Limnologica Brasiliensia, 2011, 23, 2, 164-176.

Lazia, B. Almportância da Irrigação para a Produtividade. Portal Agropecuário. 18 de maio de 2012. URL < http:// www.portalagropecuario.com.br/agricultura/irrigacao/ a-importancia-da-irrigacao-para-a-produtividade/ > Acessado em: 8 de julho de 2018.

Ribeiro, H. F. Uso de imagens digitais na diagnose do teor de nitrogênio foliar em plantas de alface. 2016. $62 \mathrm{f}$. Dissertação (Mestrado em Agronomia) - Universidade Federal do Tocantins, Gurupi.

Richards, L. A. Diagnosis and improvement of saline and alkali soil. Washington, 1954, 78, 2, 160.

Silva, A. R. A. et al. Pigmentos fotossintéticos e potencial hídrico foliar em plantas jovens de coqueiro sob estresses hídrico e salino. Revista Agroambiente On-line, 2017, 10, 4, 317-325.

Testezlaf, R. e Matsura, E. E. Engenharia de Irrigação: tubulações e acessórios. 1. ed. Campinas, SP: Faculdade de Engenharia Agrícola, UNICAMP, 2015, v. 1. 153p.

Winder, A. R.S. Lâminas De Irrigação Na Cultura Da Rúcula No Cerrado. Tese de Doutorado, 2018. 